\title{
APPLICATION OF SYSTEM-SIZE EXPANSION TO NONLINEAR REACTOR NOISE ANALYSIS
}

\author{
EHSANUR R. QUabili* \\ Department of Nuclear Engineering, The University of Michigan, Ann Arbor, Michigan 48109, U.S.A.
}

(Received for publication 11 October 1979)

\begin{abstract}
The Langevin equation with the system-size expansion has been used to study the power fluctuation in a nuclear reactor at power taking into account the nonlinearities of the point reactor kinetic equation. The delayed neutrons are neglected. Both equilibrium and nonequilibrium cases are considered. It is demonstrated that during a transient the fluctuation may be very large. A special case is solved by an alternative method, the solution of the forward stochastic equation, for the purpose of comparison. The results obtained by these two methods agreed. They also indicated that negative feedback reduces the fluctuations and this result may serve as an alternative explanation for the so called 'variance catastrophe' as referred to by Williams.
\end{abstract}

\section{INTRODUCTION}

In this paper the effect of feedback on reactor noise has been studied. Reactor noise in the presence of feedback has been studied to some extent by Harris (1958, 1964) and Karmeshu (1978). Harris, using analytical methods, solved the forward stochastic equation approximately to obtain the neutron distribution in some limiting cases. For example, when neutron multiplication approaches zero, then the neutron distribution becomes a Poisson distribution. He has shown that in the presence of feedback and an external source, one has to resort to some approximation or a priori knowledge of neutron distribution, and obtained the variance to mean ratio under the assumption that neutron distribution is gamma or normal. He has also shown that a system with a positive reactivity coefficient may be found stable when the disturbance from equilibrium is less than a certain threshold from the deterministic point of view; but may appear unstable if the system is considered to be stochastic. In a study of point reactor kinetic equations with feedback, Karmeshu (1978) used the Fokker-Planck theory assuming that the reactivity noise is white and Gaussian. In this paper the Langevin equation method is used. The implicit assumption of the method is that the basic noise sources are white. This assumption does not mean that the reactivity noise (which arises from these

* Permanent Address: Institute of Nuclear Technology, Bangladesh Atomic Energy Commission, Dacca, Bangladesh. basic noise sources) is white, due to the time constants involved in the various feedback paths.

It is well known that although the Langevin equation method is simple in stationary linear systems, it is very cumbersome in nonlinear systems because the noise source is generally nonstationary and its power spectral density is dependent on the output of the system. Van Kampen (1972) elucidated the difficulties in the application of the Langevin equation method to a nonlinear system. Akcasu (1977) has shown that the Langevin equation method with system-size expansion is, however, applicable to a nonlinear system.

In this paper we used the Langevin equation with system-size expansion to study the power fluctuation in the reactor at power taking into account the nonlinearities of the point reactor kinetic equations. A numerical example is included to demonstrate that during a transient the fluctuations may be very large. For the purpose of comparison, a special case is solved by an alternative method, the solution of the forward stochastic equation. Results obtained by these two methods agreed and indicated that negative feedback reduces the fluctuation.

\section{EVOLUTION EQUATIONS}

Let $X(t)$ and $x(t)$ be the extensive (macro) and intensive (micro) variables respectively in a uniform system of size $\Omega$. Therefore $X(t)=\Omega x(t)$. We assume that their fluctuations are described by a Markov process; and $w(x, r, t)$, the transition probability per 
unit time at $t$ from state $x$ to $x+r$ with a jump $r$, does not depend on $\Omega$ explicitly and satisfies the relation

$$
W(X, r, t)=\Omega w(x, r, t) .
$$

With the foregoing assumptions one obtains the following evolution equations for the mean and variance of the macrovariable being considered. These equations were first obtained by Kubo et al. (1973) and later by Akcasu (1977) using the system-size expansion.

$$
\begin{aligned}
\mathrm{d} y / \mathrm{d} t & =c_{1}(y, t) \\
\mathrm{d} u_{0} / \mathrm{d} t & =u_{0} c_{1}^{\prime}(y, t)+\frac{1}{2} c_{1}^{\prime \prime}(y, t) \sigma^{2}(y, t) \\
\mathrm{d} \sigma^{2} / \mathrm{d} t & =2 c_{1}^{\prime}(y, t) \sigma^{2}(t)+c_{2}(y, t) .
\end{aligned}
$$

The notations used in the above equations are explained below.

Here $y$ and $u_{0}$ are the terms in the following expansion.

$$
\langle x\rangle=y+y+\epsilon u_{0}+\epsilon^{3 / 2} u_{1},
$$

where $\epsilon=1 / \Omega$. The variance of $x(t)$ is $\epsilon \sigma(t)$ and $c_{n}(y, t)$ are the moments of the transition probability:

$$
c_{n}(y, t)=\int \mathrm{d} r r^{n} w(x, r, t)
$$

Equation (2) is known as the most probable path or the deterministic path and its solution yields deterministic results. Equation (3) describes the departure of mean from the deterministic path in the lowest order in $\epsilon$ [cf. equation (5)]. Equation (4), describing the evolution of variance, can be solved in the case of time invariant system as

$$
\sigma^{2}(y)=\sigma^{2}\left(y_{0}\right)\left[c_{1}(y) / c_{1}\left(y_{0}\right)\right]^{2}+c_{1}^{2}(y) \int_{y}^{y_{0}} \mathrm{~d} u c_{2}(u) / c_{1}^{3}(u) .
$$

\section{APPLICATION TO REACTOR NOISE}

We shall use the following definitions:

$$
\Omega=\text { volume of the reactor, }
$$

$N(t)=$ number of neutrons in the reactor,

$n(t)=N(t) / \Omega=$ neutron number density,

$r_{f}=$ fission rate per neutron,

$v=$ number of neutrons emitted per fission,

$p_{f}(v)=$ probability of obtaining $v$ neutrons in a fission,

$r_{\mathrm{c}}=$ capture rate per neutron,

$S_{0}=s_{0} \Omega=$ source event rate,

$m=$ number of neutrons emitted per source event, $p_{s}(m)=$ probability of obtaining $m$ neutrons in a source event.

We consider a simple static feedback model-the capture rate per neutron increases in proportion to the local neutron density. Thus we have

$$
r_{c}(n)=r_{c}(0)+\Gamma n,
$$

where $r_{c}(0)$ is the capture rate per neutron in the absence of feedback. Since we are considering a uniform system, we may write

$$
r_{c}(N)=r_{c}(0)+\gamma N,
$$

where $\gamma=\Gamma / \Omega$.

To simplify notation, we shall supress the argument of $r_{c}$, by expressing the total capture rate per neutron, $r_{c}(N)$, as $r_{c}+\gamma N$; where $r_{c}$ will always refer to $r_{c}(0)$. It should be noted that a positive $\gamma$ means a negative feedback and we shall consider negative feedback only. Using the above nomenclature we can express the transition probability per unit time from a state of $N$ neutrons to a state of $N+r$ neutrons as

$$
\begin{aligned}
W(N, r, t)= & N\left(r_{c}+\gamma N\right) \delta_{r,-1}+\sum p_{f}(v) r_{f} N \delta_{r, v-1} \\
& +\sum_{m} S_{0} p_{s}(m) \delta_{r, m} .
\end{aligned}
$$

Using the volume of the reactor, $\Omega$, as the size of the system in equation (2), we obtain

$$
\begin{aligned}
w(n, r, t)= & n\left(r_{c}+\Gamma n\right) \delta_{r,-1}+\sum p_{f}(v) r_{f} n \delta_{r, v-1} \\
& +\sum_{m} s_{0} p_{s}(m) \delta_{r, m} .
\end{aligned}
$$

The moments of the transition probability are given by

$$
\begin{aligned}
& c_{k}(n)=\sum_{r} r^{k} w(n, r) \\
& =(-1)^{k} n\left(r_{c}+\Gamma n\right)+r_{f} n\left\langle(v-1)^{k}\right\rangle+s_{0} m^{k} .
\end{aligned}
$$

In particular

$$
\begin{aligned}
& c_{1}(n)=-\Gamma n^{2}+\left[r_{f}\langle v\rangle-r_{f}-r_{c}\right] n+s_{0}\langle m\rangle, \\
& c_{2}(n)=\Gamma n^{2}+\left[r_{f}\left\langle(v-1)^{2}\right\rangle+r_{c}\right] n+s_{0}\left\langle m^{2}\right\rangle .
\end{aligned}
$$

We define $y$ and $u_{0}$ as [cf. equation (5)]

$$
\langle n\rangle=y+\epsilon u_{0}+O\left(\epsilon^{2}\right) \text {. }
$$

Now the most probable (deterministic) path, the evolution of the variance and the departure of the mean from the deterministic path are given by equations (2), (4) and (3), respectively.

$$
\frac{\mathrm{d}}{\mathrm{d} t} y(t)=c_{1}(y)
$$




$$
\begin{aligned}
\frac{\mathrm{d}}{\mathrm{d} t} \sigma^{2}(t) & =2 c_{1}^{\prime}(y, t) \sigma^{2}(t)+c_{2}(t) \\
\frac{\mathrm{d}}{\mathrm{d} t} u_{0} & =u_{0} c_{1}^{\prime}(y, t)+\frac{1}{2} \sigma^{2}(t) c_{1}^{\prime \prime}(y, t) .
\end{aligned}
$$

Inserting equations (12) and (13) into the above equations we get the evolution equations in reactor parameters:

$$
\begin{aligned}
\frac{\mathrm{d}}{\mathrm{d} t} y(t)= & -\Gamma y^{2}+\Delta y+q \\
\frac{\mathrm{d}}{\mathrm{d} t} \sigma^{2}(t)= & 2(\Delta-2 \Gamma y) \sigma^{2}+\Gamma y^{2} \\
& +\left[r_{f}\left\langle(v-1)^{2}\right\rangle+r_{c}\right] y+s_{0}\left\langle m^{2}\right\rangle \\
\frac{\mathrm{d}}{\mathrm{d} t} u_{0}(t)= & (\Delta-2 \Gamma y) u_{0}+\Gamma \sigma^{2}
\end{aligned}
$$

where

$$
\begin{aligned}
\Delta & =\langle v\rangle r_{f}-\left(r_{f}+r_{c}\right) \\
q & =s_{0}\langle m\rangle .
\end{aligned}
$$

(a) In equilibrium. Setting $\mathrm{d} / \mathrm{d} t=0$ in equation (18) one obtains the equilibrium values of mean, variance, etc.

$$
\begin{aligned}
y_{e}= & \frac{1}{2} \frac{\Delta}{\Gamma}\left[1 \pm\left(1+4 q \Gamma / \Delta^{2}\right)^{1 / 2}\right] \\
\sigma_{e}^{2}= & \frac{1}{2}\left[\Gamma y_{e}^{2}+\left\{r_{f}\left\langle(v-1)^{2}\right\rangle+r_{c}\right\} y_{e}+s_{0}\left\langle m^{2}\right\rangle\right] \\
& /\left(2 \Gamma y_{e}-\Delta\right) \\
u_{0 e}= & \Gamma \sigma_{e}^{2} /\left(\Delta-2 \Gamma y_{e}\right) .
\end{aligned}
$$

Since $y_{e}$ is the neutron number density we should consider only the positive equilibria. Mean neutron number density is given by [cf. equation (14)]

$$
\langle n\rangle_{e}=y_{e}+\epsilon u_{0 e}+O\left(\epsilon^{2}\right) \text {. }
$$

Various simplifications are possible depending on whether $\Gamma$ or $\Delta$ is large or small. Very small feedback will be discussed later. In a reactor at power, the external or zero-feedback reactivity, $\rho_{\text {ext }}=\Delta /\langle v\rangle r_{f}$, is positive. Of course; the net reactivity, $\rho$, is slightly negative (or zero if there is no external source) because of the induced reactivity due to the feedback; $\rho=\rho_{\text {ext }}-n \Gamma /\langle v\rangle r_{f}$. In that case

$$
\begin{aligned}
y_{e} & =\Delta / \Gamma \\
\sigma_{e}^{2} & =r_{f}\left[\langle v\rangle+\left\langle(v-1)^{2}\right\rangle-1\right] / 2 \Gamma .
\end{aligned}
$$

We notice that the variance is independent of $y_{e}$. It is interesting to note that according to equation (21b) variance is larger if the equilibrium power level $\left(\approx y_{e}\right)$ is higher; but as the equilibrium power increases (with increase of $\rho$ or $\Delta$ ) the variance saturates.

This result can also be obtained by the linear treatment. But unlike the linear treatment, the Langevin technique with system-size expansion can be used to obtain more accurate expressions, retaining the effects of nonlinearity to desired extent. To do so one has to retain higher order terms in $\epsilon$ (cf. Section 2).

(b) Nonstationary state. Let us consider a reactor operating at steady state initially (denoted by the subscript $i$ ) and at time $t=0$, the parameter $r_{c}$ has been changed from $r_{c i}$ to $r_{c e}$. This new value of $r_{c}$ will dictate a new equilibrium state of the reactor, denoted by the suffix $e$. Using equation (18), we can determine the state of the reactor as a function of time as the system relaxes from the initial (now excited) state to an equilibrium state.

The initial state $\left(y_{i}, \sigma_{i}^{2}\right.$, etc.) can be obtained simply by substituting the following into equation (21)

$$
\rho_{0 i}=\Delta_{i} /\langle v\rangle r_{f}=1-\left(r_{f}+r_{c i}\right) /\langle v\rangle r_{f}
$$

Similarly one can find the final state $\left(y_{e}, \sigma_{e}^{2}\right.$, etc.) by substituting $\rho_{0 e}$ in equation (21). Now the initial and final states, $y_{i}$ and $y_{e}$, being known, one can solve equation (15) as

$$
y(t)=y_{e}+\frac{\left(y_{i}-y_{e}\right) \mathrm{e}^{\tau t}}{1-(\Gamma / \tau)\left(y_{i}-y_{e}\right)\left(1-\mathrm{e}^{\tau t}\right)},
$$

where $\tau=\Delta-2 \Gamma y_{e}$.

It is easier to solve equation (16) in terms of $y(t)$ than in terms of $t$. One obtains

$$
\begin{aligned}
\sigma^{2}(y) & =\sigma^{2}\left(y_{i}\right)\left[\frac{c_{1}(y)}{c_{1}\left(y_{i}\right)}\right]^{2} \\
& +c_{1}^{2}(y)\left[\int_{y_{i}}^{y} \frac{-\mathrm{d} y^{\prime}}{c_{1}^{2}\left(y^{\prime}\right)}+\int_{y_{i}}^{y} \frac{\mathrm{d} y^{\prime} r_{f}\left(\left\langle v^{2}\right\rangle-\langle v\rangle\right) y^{\prime}}{c_{1}^{3}\left(y^{\prime}\right)}\right. \\
& \left.+\int_{y_{i}}^{y} \frac{\mathrm{d} y^{\prime} q\left(1+\left\langle m^{2}\right\rangle /\langle m\rangle\right)}{c_{1}^{3}\left(y^{\prime}\right)}\right]
\end{aligned}
$$

The integrations above can be performed analytically (Quabili, 1979). Once $\sigma^{2}(y)$ is obtained $u_{0}(y)$ can be obtained by solving equation (17)

$$
u_{0}(y)=u_{0}\left(y_{i}\right) \frac{c_{1}(y)}{c_{1}\left(y_{i}\right)}+\int_{y_{i}}^{y} \mathrm{~d} y^{\prime} \frac{c_{1}(y)}{c_{1}\left(y^{\prime}\right)} \frac{1}{2} \sigma^{2}\left(y^{\prime}\right) \frac{c_{1}^{\prime \prime}\left(y^{\prime}\right)}{c_{1}\left(y^{\prime}\right)}
$$




\section{A NUMERICAL EXAMPLE}

Let us consider a hypothetical reactor, described by the following parameters.

$$
\begin{aligned}
\langle v\rangle= & 2.43,\left\langle(v-1)^{2}\right\rangle=3.264, \\
\langle m\rangle= & 1,\left\langle m^{2}\right\rangle=1, \\
r_{f}= & 10^{3} \mathrm{fission}^{-1} \mathrm{~s}^{-1}, \\
s_{0}= & 100 \mathrm{n} \mathrm{cm}^{-3} \mathrm{~s}^{-1}, \\
\Omega= & 2.5 \times 10^{7} \mathrm{~cm}^{3} \\
n_{o p}= & 10^{8} \mathrm{n} \mathrm{cm}^{-3}=\text { neutron number density at } \\
& \text { operating power level, } \\
\Gamma= & 6.14 \times 10^{-8} \text { capture } \mathrm{n}^{-1} \mathrm{~s}^{-1} /\left(\mathrm{n} \mathrm{cm}^{-3}\right), \\
r_{c o}= & 1.43 \times 10^{3} \text { capture } \mathrm{n}^{-1} \mathrm{~s}^{-1}=\mathrm{a} \mathrm{reference} \\
& \text { value of } r_{c} \text { corresponding to } \rho_{0}=0 .
\end{aligned}
$$

The value of $r_{c}$, and therefore those of $\Delta$ and $\rho_{0}$ will be variable. The above value of feedback coefficient $\Gamma$, corresponds to a power reactivity coefficient $2.5 \times 10^{-5} \Delta k / k / \%$ power.

The values of mean and variance computed for different values of $\Delta$ (or equivalently $r_{c}$ ) have been tabulated in Table 1. The reactor is assumed to be at equilibrium in all three cases which may be considered to correspond to shutdown, low power and full power level.

From Table 1, one can easily notice that even at fairly low power level the variance saturates, i.e. further increase of mean neutron number does not increase the variance. One may also note that the large fluctuation at the shutdown level $\left(\sigma_{e} / y_{e} \approx 15.32\right)$ has almost disappeared at full power $\left(\sigma_{e} / y_{e} \approx 0.0196\right)$.

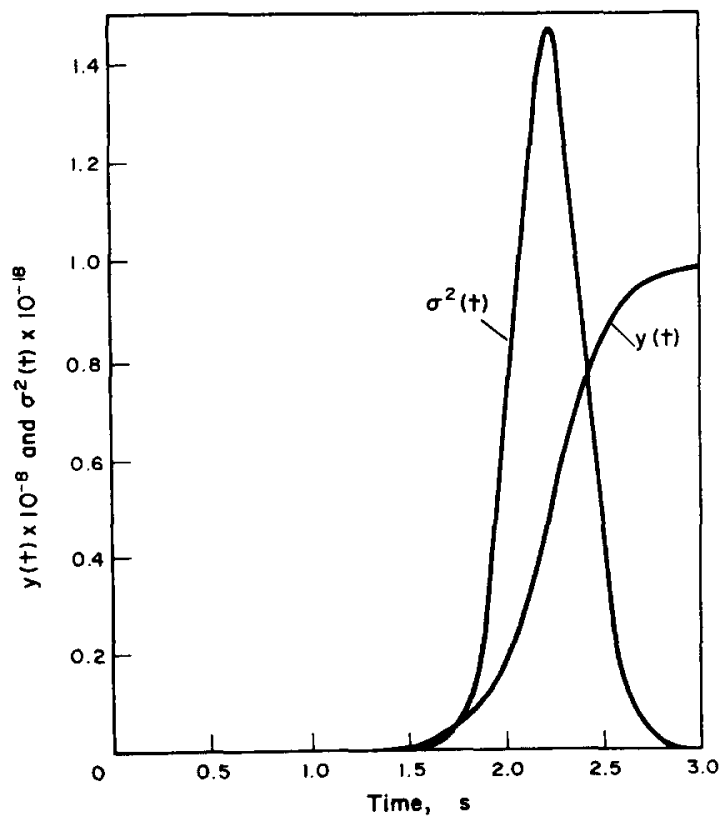

Fig. 1. Evolution of mean and variance.
Table 1. Computed statistics of neutron population

\begin{tabular}{cccc}
\hline$\Delta$ & $\rho_{\text {cxt }}(\Delta k / k)$ & $\langle n\rangle_{e} \approx y_{e}$ & $\sigma_{e}^{2}$ \\
\hline-0.1 & $-4.115 \times 10^{-5}$ & $1 \times 10^{2}$ & $2.347 \times 10^{6}$ \\
0.0614 & $2.527 \times 10^{-5}$ & $1 \times 10^{6}$ & $3.822 \times 10^{10}$ \\
6.14 & $2.527 \times 10^{-3}$ & $1 \times 10^{8}$ & $3.822 \times 10^{10}$
\end{tabular}

In the table $\epsilon u_{0 e}$ was not shown because it is too small compared to $y_{e}$ due to the large volume of the reactor.

Now we turn our attention to the nonequilibrium case. One may ask how the fluctuation will behave when the power of the reactor is changing. We shall consider an extreme case to illustrate this numerically. Let us assume that the reactor is at shutdown initially $(\Delta=-0.1)$ and the control rods are raised suddenly to a level where the reactor assumes full power $(\Delta=6.14)$. It means that the reactor is brought to full power from the shutdown state in a single instantaneous lift of the control rods or instantaneous removal of some other poison.

The evolution of mean and variance were computed from the relations (25) and (26) and are shown graphically in Fig. 1. One notices that the mean power rises very slowly at first and then rises very rapidly before it levels off at full power. The variance increases very fast during the rapid rise of power and reaches a very high value. As the power starts levelling off, variance goes down rapidly again. From this illustration it is easily noticeable that a rapid rise of the power is associated with a very large fluctuation. The maximum variance occurs approximately at half power or when the power is changing fastest and then the standard deviation is $758 \%$ of mean, very large indeed at that power level.

Of course, it is an exaggerated situation. It took only $3 \mathrm{~s}$ to reach $99 \%$ of full power from shutdown. Besides the sudden removal of the control rods, there is another reason for such a short time required. We did not consider the delayed neutrons. So this situation is overly pessimistic but the point we want to make here is clear and important-during a transient, deterministic calculations may lose its reliability. The very large variance means that in a particular event, it is highly probable that the actual power is far from the deterministically calculated power.

This conclusion is general. Any system in a severe transient should have a relatively larger fluctuation than in equilibrium. In an analysis about the magnetization of magnetic material due to cooling from above Curie temperature to below Curie temperature, Kubo et al. (1973) have predicted a very large fluctuation. In this case magnetization occurs with a 
rapid change from one state to another (random to aligned) causing a large transient.

The magnitude of the largest fluctuation depends on several factors. A larger fluctuation in $v$ and $m$ will obviously result in a larger fluctuation of power in general, i.e. both during transient and in equilibrium. In addition to that, the largest fluctuation during the transient depends on the severity of the transient (i.e. on the size of reactivity added) and on the initial mean and variance which in turn depend on the external source strength to some extent (Quabili, 1979).

\section{A SPECIAL CASE}

We intend to compare the results of the equilibrium case with those obtained by an alternative method. For that purpose we consider a special case-the feedback is very small, exactly two neutrons are emitted per fission and in a source event only one neutron is emitted. We further assume that the reactor is nearly critical. We intend to show that the results obtained in this simplified case can be reproduced by another method.

Substitution of $v=2, m=1$, and therefore, $\Delta=r_{f}-r_{c}$, in equation (21) yields

$$
\begin{aligned}
\sigma_{e}^{2} & =\left(\Gamma y_{e}^{2}+r_{c} y_{e}\right) /\left(2 \Gamma y_{e}-\Delta\right) \\
u_{0 e} & =-\Gamma\left(\Gamma y_{e}^{2}+r_{c} y_{e}\right) /\left(2 \Gamma y_{e}-\Delta\right)^{2},
\end{aligned}
$$

where we have used $-\Gamma y_{e}^{2}+\Delta y_{e}+s_{0}=0$ to eliminate $s_{0}$ [cf. equation (18a)].

The mean of neutron number density is given by equation (22) and therefore

$$
\langle n\rangle_{e}=y_{e}\left[1-\epsilon \Gamma\left(\Gamma y_{e}+r_{c}\right) \Delta^{-2}\left(1-2 \Gamma y_{e} / \Delta\right)^{-2}\right] .
$$

When the feedback is very small such that $\left|2 \Gamma y_{e} / \Delta\right| \ll 1$, we have

$$
\langle n\rangle_{e}=y_{e}\left[1-\epsilon \Gamma r_{c}\left(1+\Gamma y_{e} / r_{c}\right) \Delta^{-2}\left(1-4 \Gamma y_{e} / \Delta\right)\right] .
$$

Also for small $\Gamma$, one obtains from equation (21a)

$$
y_{e}=n_{0}\left(1-s_{0} \Gamma / \Delta^{2}\right),
$$

where $n_{0}=-s_{0} / \Delta$ is the neutron number density in the absence of feedback. Combining above two equations we obtain

$$
\langle n\rangle_{e}=n_{0}\left[1-\left(s_{0} \Omega / r_{c}+1\right)\left(\epsilon \Gamma / r_{c}\right)\left(1-r_{f} / r_{c}\right)^{-2}\right],
$$

where we have kept terms in lowest order in $\Gamma$ and used $\epsilon \Omega=1$. Now using the near criticality assumption, i.e. $r_{f} \approx r_{c}$ and reverting to $N, S_{0}$, etc. (extensive variables) from $n, s_{0}$, etc. (intensive variables) we get

$$
\langle N\rangle_{e}=N_{0}\left[1-\left(1+S_{0} / r_{f}\right)\left(1-r_{f} / r_{c}\right)^{-2} \gamma / r_{c}\right] .
$$

The variance to mean ratio at equilibrium, $\eta_{e}$, can be obtained from equation (28a) as

$$
\eta_{e}=\sigma_{e}^{2} / y_{e}=-r_{c}\left(1+\Gamma y_{e} / r_{c}\right)\left(1-2 \Gamma y_{e} / \Delta\right)^{-1} \Delta^{-1} .
$$

As before, expanding $\left(1-2 \Gamma y_{e} / \Delta\right)^{-1}$ and keeping terms in the lowest order in $\Gamma$, we get

$$
\eta=\left(1-r_{f} / r_{c}\right)^{-1}\left(1+\Gamma y_{e} / r_{c}+2 \Gamma y_{e} / \Delta\right) .
$$

Approximating $y_{e}$ in the above expression by $n_{0}$ and using the near criticality assumption, i.e. $r_{f} \approx r_{c}$, we obtain

$$
\eta=\frac{1}{1-r_{f} / r_{c}}\left(1-2 S_{0} \gamma / \Delta^{2}\right) .
$$

The probability generating function associated with a random variable $x$ is defined by

$$
g(\mu, t)=\sum_{x} \mu^{x} P(x, t)
$$

If $x$ happens to be continuous, the summation should be replaced by an integration. The moments of $x$ can be obtained from the derivatives of $g(\mu, t)$; e.g.

$$
\langle x\rangle=\partial g /\left.\partial \mu\right|_{\mu=1} ;\left\langle x^{2}\right\rangle=\left.\left(\partial g / \partial \mu+\partial^{2} g / \partial \mu^{2}\right)\right|_{\mu=1} .
$$

Inserting equation (9) in equation (1) we obtain the Master equation satisfied by $P(N, t)$ :

$$
\begin{aligned}
\partial / \partial t P(N, t)= & -P(N, t)\left(r_{f} N+r_{c} N+\gamma N^{2}+S_{0}\right) \\
& +P(N+1, t)(N+1) r_{c}+\gamma(N+1)^{2} \\
& +\sum_{v} P(N+1-v, t) p_{f}(v) r_{f} \\
& +\sum_{m} P(N-m, t) p_{s}(m) S_{0}
\end{aligned}
$$

We multiply equation (35) by $z^{N}$, sum over all $N$ and use the following probability generating functions for neutron, fission and source distributions, respectively,

$$
\begin{gathered}
g(z, t)=\sum_{N} z^{N} P(N, t), g_{f}(z)=\sum_{N} z^{N} p_{f}(N) \\
g_{s}(z)=\sum_{N} z^{N} p_{s}(N)
\end{gathered}
$$

and obtain the following forward stochastic equation

$$
\begin{aligned}
\frac{\partial}{\partial t} g(z, t)= & {\left[r_{f}\left\{g_{f}(z)-z\right\}+r_{c}(1-z)\right] \frac{\partial}{\partial t} g(z, t) } \\
& +S_{0}\left\{g_{s}(z)-1\right\} g(z, t) \\
& +\gamma(1-z) \frac{\partial}{\partial z} z \frac{\partial}{\partial z} g(z, t) .
\end{aligned}
$$


A general solution of equation (37) is difficult to obtain and here we consider the same special casenear critical steady state system with $v=2$ and $m=1$ exactly. In this case we have $\partial / \partial t=0, g_{f}(z)=z^{2}$ and $g_{s}(z)=z ;$ and equation (37) reduces to

$$
\gamma z \mathrm{~d}^{2} g / \mathrm{d} z^{2}+\left(r_{\mathrm{c}}+\gamma-r_{f} z\right) \partial g / \partial z-S_{0} g(z)=0 .
$$

The solution is given by

$$
g\left(r_{f} z / \gamma\right)=M\left(a, b ; r_{f} z / \gamma\right) / M\left(a, b ; r_{f} / \gamma\right),
$$

where

$$
a=S_{0} / r_{f} ; b=1+r_{c} / \gamma .
$$

The confluent hypergeometric function used in the above solution is defined by

$$
M(u, v ; \mu)=1+\frac{u \mu}{v}+\frac{(u)_{2} \mu^{2}}{(v)_{2} 2 !}+\cdots,
$$

where

$$
(v)_{n}=(v+1) \ldots(v+n-1) .
$$

We shall use the following properties of confluent hypergeometric function:

$$
\frac{\mathrm{d}}{\mathrm{d} \mu} M(u, v ; \mu)=\frac{u}{v} M(1+u, 1+v ; \mu) .
$$

If $u$ is bounded and $v$ and $\mu$ simultaneously go to infinity such that

$$
\mu<v, \mu=\lambda v ; 0<\lambda<1-\xi \quad(\xi<0),
$$

$M(u, v ; \mu)$

$$
=(1-\lambda)^{-u}\left[1-\frac{u(1+u)}{2 v}\left(\frac{\lambda}{1-\lambda}\right)^{2}+O\left(|v|^{-2}\right)\right] .
$$

Using equations (34), (39) and (41) we obtain

$$
\begin{aligned}
\langle N\rangle_{e}=\frac{r_{f}}{\gamma} \frac{a}{b} \frac{M\left(1+a, 1+b ; r_{f} / \gamma\right)}{M\left(a, b ; r_{f} / \gamma\right)} \\
\left\langle N^{2}\right\rangle=\left(r_{f} / \gamma\right)^{2} \frac{a(1+a)}{b(1+b)} \frac{M\left(2+a, 2+b ; r_{f} / \gamma\right)}{M\left(a, b ; r_{f} / \gamma\right)} \\
+\langle N\rangle_{e} .
\end{aligned}
$$

Now, as $\gamma \rightarrow 0$, equation (42a) is satisfied and therefore equation (42b) can be used to reduce equation (43a) into

$$
\begin{aligned}
\langle N\rangle_{e} & =\frac{a}{b} \frac{r_{f}}{\gamma} \frac{\left(1-r_{f} / \gamma b\right)^{-(1+a)}}{\left(1-r_{f} / \gamma b\right)^{-a}} \\
& \times \frac{1-(1+a)(2+a) /\left(2 b \Lambda^{2}\right)}{1-a(1+a) /\left(2 b \Lambda^{2}\right)},
\end{aligned}
$$

where we have used

$$
M\left(1+a, 1+b ; r_{f} / \gamma\right) \approx M\left(1+a, b ; r_{f} / \gamma\right)
$$

(justified because $b \rightarrow \infty$ ) and

$$
\Lambda=\frac{r_{f} / \gamma b}{1-r_{f} / \gamma b}
$$

As $\gamma \rightarrow 0$, we also have

$$
\Lambda=r_{f} / \Delta ; a \Lambda=N_{0}
$$

and

$$
(1+a)(2+a) /\left(2 b \Lambda^{2}\right) \ll 1 .
$$

Therefore we can expand the denominator in equation (44), keep the lowest order terms in $\Lambda^{2} / b$ and approximate $b$ by $r_{c} / \gamma$ to obtain

$$
\langle N\rangle_{e}=N_{0}\left[1-(1+a) \Lambda^{2} \gamma / r_{c}\right] .
$$

Using $r_{f} \approx r_{c}$ we reproduce equation (31).

Following an identical procedure we get from equation $(43 \mathrm{~b})$

$$
\begin{aligned}
\frac{\left\langle N^{2}\right\rangle_{e}}{\langle N\rangle_{e}} & =1+\frac{(1+a) r_{f} / \gamma}{b} \frac{M\left(2+a, b ; r_{f} / \gamma\right)}{M\left(1+a, b ; r_{f} / \gamma\right)} \\
& =1+\left(\Lambda+N_{0}\right)\left(1-(2+a) \Lambda^{2} \gamma / r_{c}\right) .
\end{aligned}
$$

Therefore

$$
\begin{aligned}
\eta_{e} & =\left\langle N^{2}\right\rangle_{e} /\langle N\rangle_{e}-\langle N\rangle_{e} \\
& =1+\frac{r_{f}}{\Delta}-2\left(\frac{r_{f}}{\Delta}\right)^{3}\left(1+S_{0} / r_{f}\right) \gamma / r_{c},
\end{aligned}
$$

where we have used equations (48), (46) and (39b). Rearranging and using $r_{f} \approx r_{c}$ we find

$$
\eta_{e}=\frac{1}{1-r_{f} / r_{c}}\left[1-2 \gamma r_{f}\left(1+S_{0} / r_{f}\right) / \Delta^{2}\right] .
$$

Now if we keep $s_{0}$ and $r_{f}$ constant and make $\Omega$ sufficiently large then we have $S_{0} / r_{f} \gg 1$ and equation (50) reduces to equation (32) exactly.

\section{DISCUSSION AND CONCLUSIONS}

It was found that the reactor noise analysis using the Langevin equation with system-size expansion is straightforward. In equilibrium, the results obtained in the lowest order in $\epsilon$ is the same as those obtained by the linear treatment. But unlike linear treatment, using this method one can pursue more accurate analysis (retaining the effects of nonlinearity) by using a higher order description (cf. Section 2). Keeping higher order terms in $\epsilon$ is particularly important when the size of the system is small. However, the 
more important aspect of this method is the relative ease of studying the fluctuations in a nonstainary state.

The solution of the forward stochastic equation in the special case compares favorably with the Langevin equation with system-size expansion. The case considered is unrealistic so far as the nuclear reactors are concerned, because $v$ was assumed to be 2 . Such assumption was necessary even in equilibrium so that a reasonably simple solution for equation (37) can be found. It may be noted that the solution (39) may not prove to be a useful analytical solution always, because of the difficulties involved in the evaluation of confluent hypergeometric functions. Despite these facts, the solution (39) may be interesting to a biologist because this is an exact solution and $v=2$ describes the cell multiplication exactly.

It was observed that in equilibrium, negative feedback reduces the variance to mean ratio, i.e. arrests fluctuation to some extent. Although it may be observed from equation (21), it is transparent in equation (32) or (50). We observe that even in the absence of a source (critical reactor) the system has finite variance with a nonzero mean. It has been long established that a critical system cannot have both mean and variance finite. That is if the mean is finite, the variance goes to infinity and if the variance is finite, the mean goes to zero with increase of time. The existence of critical systems in practice is popularly explained as follows. A critical system is never 'critical' because some external source, no matter how small, is always present. Therefore, the so called critical system is always 'subcritical'. As mentioned by Williams (1974), to some this explanation is not highly convincing and they maintain that possibly some feedback phenomenon prevents the "variance catastrophe' to happen. Here we find feedback indeed does so.

Finally, we have observed that during transients fluctuations may be very large. In normal reactor operations the statistical fluctuations are of little importance, but in an accident condition, the transients may be large causing severe fluctuations which may reduce the significance of the deterministic calculations.

Acknowledgements-The author expresses his deep gratitude to Professor A. Ziya Akcasu for his help in all stages in this investigation. The Phoenix Memorial Project of the University of Michigan provided financial help for this investigation.

\section{REFERENCES}

Akcasu A. Ziya (1977) J. Stat. Phys. 16, 33.

Harris D. R. (1958) WAPD-TM-190.

Harris D. R. et al. (1964) Naval Reactor Physics Handbook, Vol. 1, pp. 1010-1085. (Ed. Radkowsky A.) USAEC.

Karmeshu (1978) Ann. nucl. Energy 5, 21.

Kubo R. et al. (1973) J. Stat. Phys. 9, 51.

Quabili E. R. (1979) Ph.D. Thesis, University of Michigan, Ann Arbor, Michigan.

Van Kampen N. G. (1961) J. Math. Phys. 2, 592.

Van Kampen N. G. (1972) Irreversibility in the Many Body Problem, pp. 369-386. (Ed. Biel J. and Rae J.). Plenum Press, New York.

Williams M. M. R. (1974) Random Processes in Nuclear Reactors. Pergamon Press, Oxford. 